\title{
Die for the community: an overview of programmed cell death in bacteria
}

\author{
N Allocati, ${ }^{*, 1}$, M Masulli ${ }^{1}$, C Di llio ${ }^{1,2}$ and V De Laurenzi ${ }^{1,2,3}$
}

Programmed cell death is a process known to have a crucial role in many aspects of eukaryotes physiology and is clearly essential to their life. As a consequence, the underlying molecular mechanisms have been extensively studied in eukaryotes and we now know that different signalling pathways leading to functionally and morphologically different forms of death exist in these organisms. Similarly, mono-cellular organism can activate signalling pathways leading to death of a number of cells within a colony. The reason why a single-cell organism would activate a program leading to its death is apparently counterintuitive and probably for this reason cell death in prokaryotes has received a lot less attention in the past years. However, as summarized in this review there are many reasons leading to prokaryotic cell death, for the benefit of the colony. Indeed, single-celled organism can greatly benefit from multicellular organization. Within this forms of organization, regulation of death becomes an important issue, contributing to important processes such as: stress response, development, genetic transformation, and biofilm formation.

Cell Death and Disease (2015) 6, e1609; doi:10.1038/cddis.2014.570; published online 22 January 2015

\section{Facts}

- Programmed cell death $(P C D)$ is not restricted to multicellular organisms.

- Different forms of death have been described in bacteria.

- Bacteria are able to induce cell death in a part of the population in response to adverse conditions to favour the survival of the community.

- PCD has a crucial role in the development of a bacterial population.

\section{Open Questions}

- Do bacterial and eukaryotic PCD have a common origin?

- Are there additional unknown forms of death in prokaryotes?

- Can we exploit knowledge on molecular mechanisms of bacterial PCD to develop alternative therapeutic strategies against multiresistant pathogens?

In eukaryotes, death is essential for life. Indeed, PCD is fundamental for many processes, including: embryogenesis, tissue homeostasis, and immune and stress response. ${ }^{1-4}$ Therefore it has been assumed that PCD had emerged with multicellularity, being absent in unicellular organisms.,5,6 However, it is now clear that PCD also occurs in unicellular eukaryotes and bacteria and is therefore present in all life kingdoms. ${ }^{3,7-14}$ Microorganisms are social organisms and many of them can switch from unicellular to multicellular organization such as microbial colonies, biofilms, and aggregates. ${ }^{15}$ Multicellular organization offers many advantages, such as increased protection against hostile environments, increased genetic diversity, and improved food availability. ${ }^{16-18}$ A bacterial population - acting as a multicellular organism - would use PCD programmes to sacrifice part of the colony to sustain the survival of the remaining cells. ${ }^{7,19}$

Bacteria communicate through a process named quorum sensing (QS) to modulate gene expression in response to changes in cell density. ${ }^{20}$ The increase of cell density, resulting in the accumulation of the signalling molecules in the environment, activates the response. QS allows bacteria to synchronize the gene expression pattern of the colony and thus behave like a multicellular organism and is involved in several bacterial processes such as biofilm, sporulation, production of virulence factors, and competence for DNA uptake. ${ }^{20-22}$ Clearly QS may result in activation of death pathways to control colony size as shown by some examples discussed in this review.

In multicellular eukaryotes, death comes in many flavours and the term PCD describes a number of biological processes that differ for morphological characteristics as well as for the underlying molecular pathways. Many of these have now been well characterized at the molecular level, and recently, the Nomenclature Committee on Cell Death has proposed a new classification based on biochemical features rather than morphological characteristics. ${ }^{23-26}$

Similarly, different forms of PCD can be observed in prokaryotes but these are in general less characterized. ${ }^{7,27-30}$

'Department of Experimental and Clinical Sciences, University "G. d'Annunzio", Chieti I-66013, Italy; ${ }^{2}$ Center of Excellence on Aging (Ce.S.I.), University "G. d'Annunzio", Chieti I-66013, Italy and ${ }^{3}$ BIOUNIVERSA srl, University of Salerno, Fisciano (SA), Italy

*Corresponding author: N Allocati, Department of Experimental and Clinical Sciences, University “G. d'Annunzio", Via dei Vestini, Chieti I-66013, Italy. Tel: +39 0871 355 4807; Fax: +39 0871355 4800; E-mail: allocati@ unich.it

Abbreviations: $P C D$, programmed cell death; $P G$, peptidoglycan; TA, toxin-antitoxin; $Q S$, quorum sensing

Received 16.6.14; revised 25.11.14; accepted 01.12.14; Edited by G Melino 
As an example, PCD has been demonstrated experimentally by synthetic-biology approaches in E. coli cells, outlining the benefits of altruistic death under stressing conditions. ${ }^{31}$

Bacteria are able to control cell death under several stressful conditions, such as high temperature, amino-acid starvation, and antibiotic treatment. ${ }^{29,32,33}$ Indeed, the well-known toxinantitoxin (TA) system has a role in cell survival under cellular damage or several stressing conditions, resulting in either cell growth arrest or a form of cell death similar to apoptosis. ${ }^{29}$ Furthermore, it appears that PCD has a key role in various developmental processes, such as: autolysis of bacterial cells during the formation of fruiting body in myxobacteria, ${ }^{34}$ hyphae differentiation in streptomyces, ${ }^{35}$ sporulation of bacilli, and DNA transformation in streptococci. ${ }^{36}$

In this review, we try to give a comprehensive overview of the different forms of death described in bacteria.

\section{PCD in Stress Response}

A bacterial community can induce death in a part of the population in response to various stress conditions to favour the survival of the colony, including: oxidative stress, radiation exposure, nutrient deprivation, phage infections, and many others. In most of these cases, PCD is induced through TA mechanisms.

TA systems. TA systems are involved in several processes, such as formation of persistent cells, plasmid stabilization, peptidoglycan (PG) synthesis, resistance to bacteriophages and antibiotics, inhibition of macromolecule, and biofilm formation. ${ }^{29,37,38}$ Most bacteria and archaea contain TA loci in their genomes, often in multiple copies, present in both extra chromosomal and chromosomal DNA. ${ }^{29}$ TA loci are typically organized in operons with two genes constitutively encoding a stable toxin that disrupts an essential cellular process and an unstable antitoxin that prevents its toxicity. ${ }^{29,32,39}$ Toxins and antitoxins form a stable complex inhibiting the toxin activity under normal growth conditions. In case of cellular damage or stress conditions, antitoxins are degraded, allowing the free toxin to bind its cellular target. To date, three types of TA systems are known and are classified on the basis of the function of the antitoxin and the composition of the TA system. ${ }^{29,32,39}$ More recently, two additional types of TA systems have been identified; ${ }^{32,40}$ however, it is still questionable if their distinguishing features justify a new classification.

In type I TA systems, toxin gene expression is regulated by an antisense RNA (RNA-antitoxin) transcribed from a gene adjacent to the toxin gene but in reverse orientation. ${ }^{29}$ Type I TA systems - that is, the hok/sok system - mediate plasmid maintenance, inducing death in plasmid-free cells. ${ }^{41,42}$ Annealing of the antisense RNA to the toxin mRNA prevents toxin translation (Figure 1a). When the plasmid is lost following cell division, the antitoxin (an unstable RNA) is rapidly degraded allowing translation of the more stable toxin mRNA thus leading to cell death. The molecular mechanism through which these toxins kill the cell is still unclear but it is probably associated with membrane depolarization and increased membrane permeability. ${ }^{41,42}$

In type II TA systems, two genes, codifying for two small proteins, are organized in an operon and are regulated at the transcriptional level. The two proteins form an TA stable complex that inhibits the harmful effects of the toxin ${ }^{29}$ (Figure 1b). Following cell damage, stress-induced proteases digest the antitoxin freeing the toxin leading to inhibition of cell growth or to cell death. ${ }^{29}$ Several type II TA systems have been identified and their biological role characterized. ${ }^{37,43,44}$ The first type II TA system described was the mazE/mazF module that is extensively diffused among bacteria and is based on the activity of the toxin MazF, a ribosome-independent mRNA interferase. ${ }^{45}$ This enzyme is a specific endoribonuclease that, when induced, cleaves cellular mRNAs inhibiting cell growth. In E. coli, the toxin is activated in response to several types of stress conditions, such as: high temperature, aminoacid starvation, oxidative stress, and antibacterial pressure, resulting in cell growth arrest and eventually in PCD. ${ }^{37,46}$ Antibacterials that inhibit RNA and/or protein synthesis affect mazEF expression. ${ }^{19}$ The consequent drastic reduction of cellular concentration of MazE releases the MazF toxin triggering cell death. ${ }^{19}$ The mazEF module is also involved in bacterial persistence (see below). In Myxococcus xanthus, an MazF homologue has a crucial role in the fruiting body formation (see below). It has been recently reported that mazEF-mediated cell death in $E$. coli is a population phenomenon requiring the presence of a QS factor called extracellular death factor (EDF). ${ }^{47}$ EDF is a linear pentapeptide that specifically affects the toxin significantly amplifying its enzymatic activity. Similar results have been obtained for the ChpBK toxin of the type II TA system chpBIK in E. coli. ${ }^{47}$ More recently, EDFs have been also found in the Gram-positive Bacillus subtilis and in the Gram-negative Pseudomonas aeruginosa. ${ }^{48}$ EDFs of $B$. subtilis and $P$. aeruginosa have been shown to be able to trigger $E$. coli mazEF, providing the first example of a QS factors participating in interspecies bacterial cell death. ${ }^{48}$ Therefore, it has been proposed that the induction of the altruistic suicide mechanism by EDFs may be used from a bacterial species - under stressing conditions - to kill another in a mixed population. ${ }^{48}$ EDFs have the potential to be exploited to generate a new class of antibiotics that trigger death from outside the bacterial cells. ${ }^{49}$

Members of the epsilon/zeta TA family are also involved in the virulence of several human pathogens. ${ }^{50}$ An example is the PezA/PezT system (pneumococcal epsilon zeta) in Streptococcus pneumoniae. The PezT toxin phosphorylates the PG precursor uridine diphosphate- $N$-acetylglucosamine, causing the inhibition of MurA. This enzyme catalyses one of the first steps of PG synthesis, therefore its block results in autolysis of rapidly growing bacteria. As a consequence, the pore-forming toxin pneumolysin, a major virulence factor that accelerates infection progression, is released. ${ }^{50}$

Other type II TA modules, that is, hipBA, mazEF, and $m q s R A$, are involved in bacterial persistence. ${ }^{29,51}$ In this case, toxins, rather than causing cell death, induce a quasi-dormant cell state. This is characterized by the presence in a bacterial population of a small group of slowly growing cells - called persisters - highly tolerant to a number of environmental insults such as antibiotics, to which the numerically prevalent exponentially growing cells are sensitive. ${ }^{51}$ Usually this state is induced by toxins released by stress-induced proteases. One of these is the HipA (high persistence) toxin which together with the corresponding HipB antitoxin is encoded by 

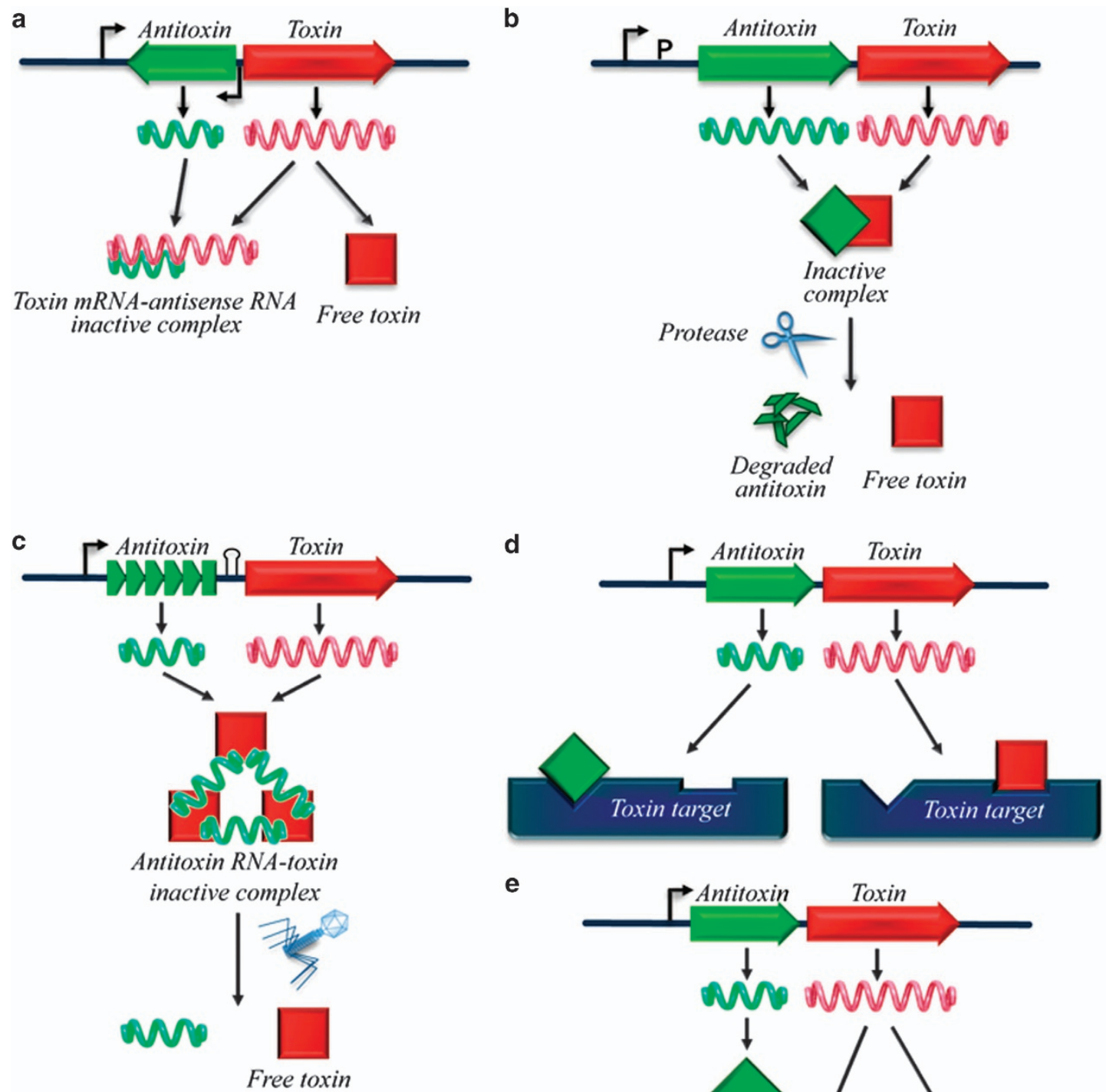

e



Degraded toxin mRNA

Figure 1 Bacterial TA systems. Bacterial TA systems are composed of a toxin and an antitoxin that neutralizes its effect. They are classified on the basis of the function of the antitoxin and the composition of the TA module. In all the TA system, in response to various stimuli the antitoxin is degraded allowing the toxin to act on its target generally resulting in either bacterial growth arrest or cell death. (a) Type I: antisense RNA antitoxin binds to the mRNA encoding for the toxin blocking its translation. Loss of the unstable antisense mRNA allows transcription of the sense strand. (b) Type II: toxin and antitoxin generally transcribed in the same operon form an inactive complex. Protease-dependent degradation of the antitoxin in response to stress frees the active toxin. (c) Type III: antitoxin RNA binds and inactivates to the toxin protein. (d) Type IV: the antitoxin prevents the effect of the toxin by binding the toxin target. Again in response to stress, degradation of the antitoxin allows binding of the toxin to its target. (e) Type V: the antitoxin binds and cleaves the mRNA encoding for the toxin

hipBA locus. Inhibition of cell growth and stimulation of persisters formation requires HipA serine kinase activity, ${ }^{52}$ which supposedly exerts its effect through phosphorylation and consequent inhibition of the elongation factor Tu (EF-Tu), essential for the elongation phase of protein synthesis. ${ }^{52}$ Finally, type II TA systems also have a role in biofilm formation as described later. ${ }^{50,53,54}$

Type III TA systems consist of an RNA antitoxin molecule that inhibits toxins by the formation of a RNA-protein complex. ${ }^{55,56}$ One example is the ToxIN system, one of the many mechanisms that bacteria use to withstand bacteriophage infection. ${ }^{57}$ In this system, altruistic suicide of an infected cell reduces phage infection within the population. The abortive infection system, ToxIN, first identified in the phytopathogen Erwinia carotovora and later in several genera of Gram-negative and Gram-positive bacteria, functions as a typical type III TA system via a novel RNA-protein mechanism $^{55,58,59}$ (Figure 1c). toxN gene - codifying an 
endoribonuclease - is preceded by a short palindromic repeat that acts as a transcriptional terminator (stem-loop), regulating the relative levels of both antitoxin RNA and toxin transcript. Upstream there is the antitoxin (Toxl), a repetitive array containing 5.5 tandem repeats of a $36 \mathrm{nt}$ sequence. Each $36 \mathrm{nt}$ ToxI RNA repeat can inhibit the activity of ToxN. ToxIN complex is composed of a heterohexameric triangular assembly of three ToxN proteins interlaced by three ToxI RNAs. ${ }^{59}$ ToxI RNA antitoxin interacts directly with ToxN inhibiting its toxic activity instead of preventing its expression. During phage infection, the Toxl:ToxN ratio changes, probably due to alterations in host transcription or translation or the degradation of bacterial DNA, resulting in the release of the active toxin that consequently cleaves cellular and phage RNAs. ${ }^{55,60}$

In type IV TA systems, the antitoxin does not inhibit the toxin through direct binding but neutralizes its toxicity by stabilizing the toxin target proteins ${ }^{61}$ (Figure 1d). A prototype of these systems is the YeeU/YeeV (CbeA/CbtA) module, involved in the regulation of cytoskeletal proteins and as a consequence of cellular morphology changes and division. The toxin CbtA inhibits the polymerization of the cytoskeletal proteins MreB and FtsZ. The antitoxin YeeU causes the opposite effect, interacting with two proteins that promote the assembly of the filaments. Under stress conditions, YeeU is degraded allowing CbtA to bind its targets resulting in altered cytoskeleton and inhibition of cell division. ${ }^{61}$ Finally and more recently, type $\mathrm{V}$ systems have been described in which the antitoxin acts as an endoribonuclease that degrades the mRNA coding for toxin mRNA (Figure 1e). E. coli uses such a system, where endoribonuclease GhoS specifically degrades the mRNA coding for the membrane lytic peptide GhoT by cleaving its mRNA. ${ }^{62}$

Holin-endolysin system. dsDNA bacteriophages induce cell death through a holin-endolysin system, ${ }^{63,64}$ at the end of the lytic cycle, to release new bacteriophage particles. Holins are small proteins that accumulate in the host membrane where they induce hole formation allowing endolysins - enzymes with PG hydrolase activity - to reach and degrade the PG leading to cell lysis. ${ }^{63}$ Antiholins finely tune the system by inhibiting holins, thus regulating the accurate timing of endolysin release.

\section{Role of PCD in Bacterial Development}

PCD is essential for the proper development of a bacterial population and contributes to it in many ways: providing nutrients to the sibling cells; releasing components of the biofilm matrix such as DNA; and promoting special aspects of the life cycle and of biofilm development.

Cannibalism and autolysis during sporulation. Endospores are metabolically dormant and resistant bodies, produced by Bacillus and Clostridium and other related species formed in response to high cell density and to severe external stress - such as nutritional stress for Bacillus spp. and acidification for Clostridia spp. Cell division during sporulation produces two different cells: a smaller one, the prespore which evolves into the spore and a bigger one, the mother cell, which is essential for spore formation (Figure 2a).
When its maturation is completed the spore is released, and this requires PCD by autolysis of the mother cell. This phenomenon involves the activity of specific enzymes, PG hydrolases (see Box 1). In Bacillus subtilis, two PG hydrolases, LytC and CwIC, are present in large amounts and are responsible for the hydrolysis of the mother cell $P G$ allowing the release of the mature endospore. ${ }^{65}$

During the early events of sporulation, before the process becomes irreversible, a part of $B$. subtilis cells can produce extracellular factors to induce a death program and 'cannibalize' sister cells that release nutrients causing delay or complete block of the sporulation (Figure 2a). The process becomes irreversible when the cells committed to sporulate form an asymmetric polar septum. ${ }^{66}$

Cannibalism offers various advantages to the bacterial community. Sporulation process requires a lot of energy and time to be completed. Furthermore, in the availability of nutrients, spores do not return to active growth as efficiently as vegetative cells. Thus, in a mixed community, where most of the bacteria are non-sporulating, the germination of the spores could represent a disadvantage. In the absence of cannibalism, all bacteria sporulate at the same time, and therefore returning to vegetative life is slower and more complex. Therefore, cannibalism helps to maintain in the population a small percentage of spores and a higher number of growing cells, during the long-term stationary phase of growth. ${ }^{66}$ Moreover, in the mixed community where $B$. subtilis lives and competes with other species, bacteria not only gain nutritional benefits through consuming their siblings but also may eliminate potential competitors and predators.

The molecular mechanism of 'cannibalism' involves two gene clusters, $s k f$ and $s d p$, controlled by the Spo0A regulon, the main regulator of sporulation. ${ }^{36,66}$ Although the killing factor produced by $s k f A-H$ operon has not been completely characterized, it appears that its activity is similar to that of bacteriocins. In the second cluster of genes, the $s d p C$ gene produces a peptide toxin, SDP, that acts on the proton motive force of the adjacent cells by inhibiting mobility and secretion of proteins and inducing cell lysis. ${ }^{67}$ SDP toxin appears to have a role also in the defence towards invading bacteria. ${ }^{67}$ In fact, the collapse of proton motive force inhibits flagellar motility in several species preventing their ability to invade the colony. Furthermore, it has been suggested that SDP can inhibit biofilm formation by surrounding bacterial species, decreasing competition in the ecological niche. Finally, bacteria with compromised proton motive force undergo autolysis, providing an additional free source of nutrients for the colony. ${ }^{67}$

Fratricide behaviour during genetic transformation. In some cases, bacteria, in response to environmental signals, can induce a death program in neighbouring cells in order to uptake their genetic material (Figure 2b). Indeed in S. pneumoniae, cells competent for natural genetic transformation produce toxins that will kill non-competent sisters and uptake their DNA and incorporate it into their DNA by recombination. ${ }^{36,68}$ In these bacteria, competence is induced by the competence-stimulating peptide (CSP) that triggers the process of transformation by interacting with its receptor ComD. Several killing factors are involved in the lysis of pneumococcal cells. The PG hydrolase $\mathrm{CbpD}$ is the first 


\section{Cannibalism in Bacillus subtilis}

Nutrient limitation
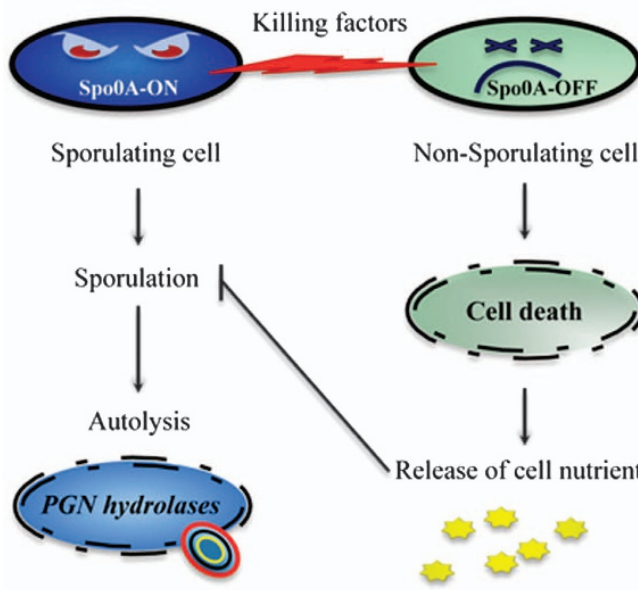

C

\section{Life cycle of Myxococcus xanthus}

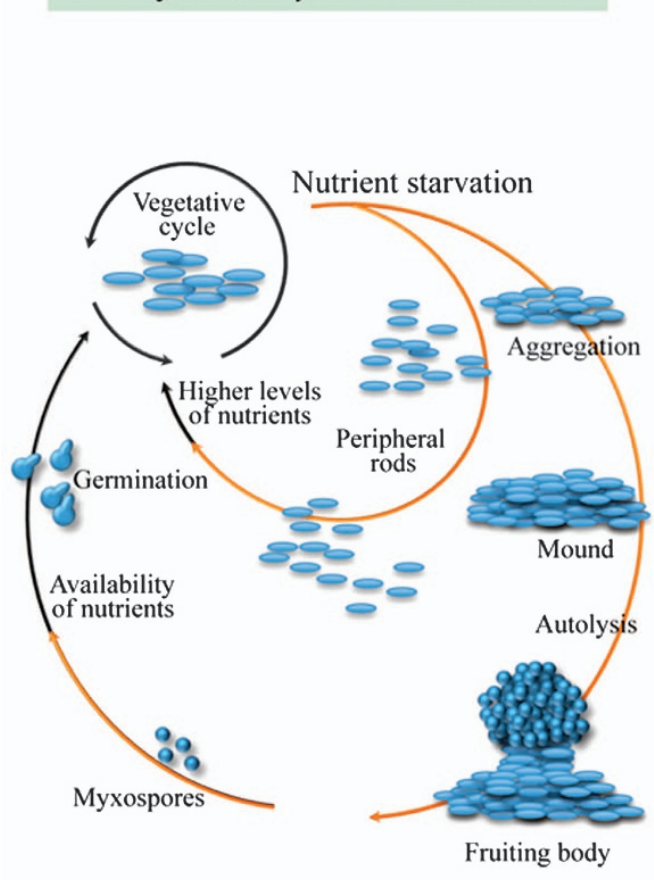

Fratricide in Streptococcus pneumonie

Environmental signal

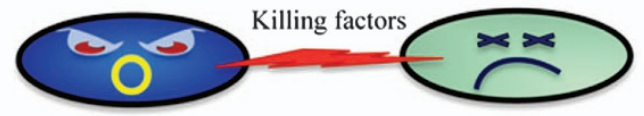

Competent cell<smiles>CCCCC</smiles>

Genetic transformation
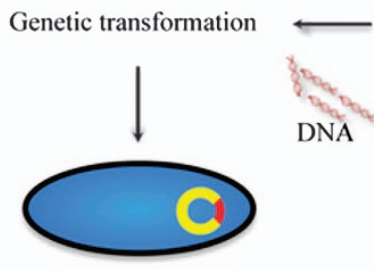

Non-Competent cell

d

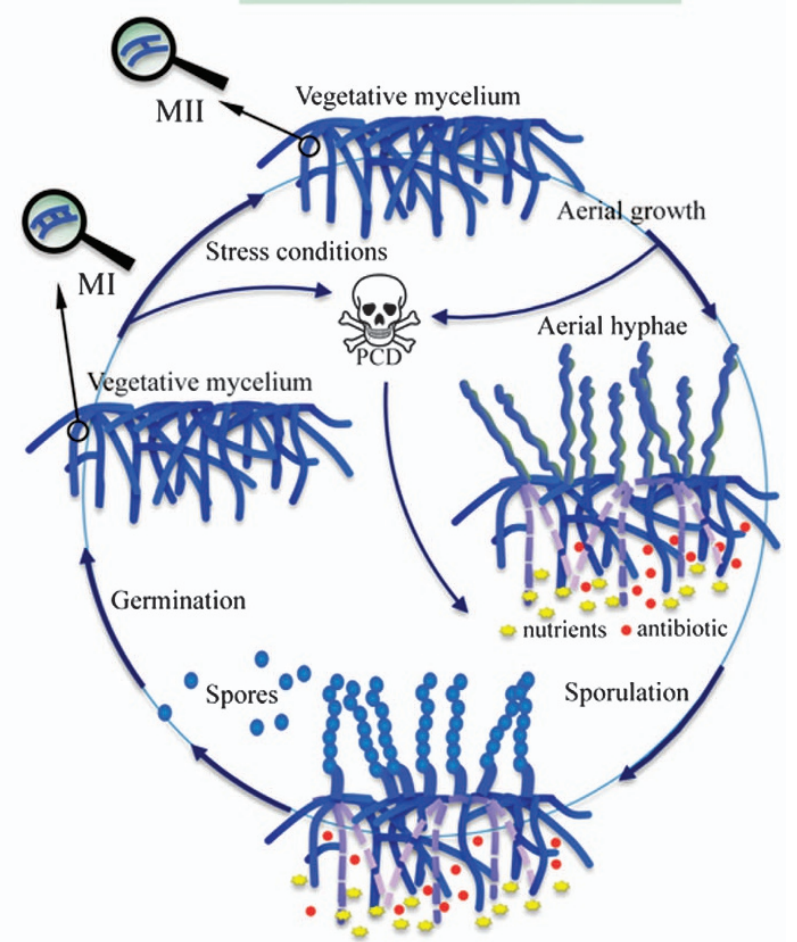

Figure 2 Examples of bacterial programmed cell death. (a) Upon nutrient limitation Bacillus subtilis undergoes two fates: sporulation or non-sporulation. Death of non-sporulating cells results in nutrient release that supports sporulation. Moreover, the mother cell in the sporulating population undergoes PCD to release the mature spore. (b) Competent Streptococcus pneumonaie cells induce death of non-competent cells in order to incorporate their DNA for genetic transformation. (c) Upon nutrient starvation of Myxococcus xanthus, while a small percentage of cells remains undifferentiated and forms the peripheral rods, the majority of cells undergoes fruiting body formation. During this process, a large number of cells is lysed in order to release nutrients for the remaining cells that will differentiate into mixospores. When nutrients are available, a new colony rises from proliferation of peripheral rods cells and germination of myxospores. (d) Under stressing conditions, a part of Ml cells is subjected to PCD while the remaining viable cells differentiate into MII cells. In the second phase of PCD, a part of MII cells dies releasing nutrients to feed the aerial mycelium, which is developed from branches of the remaining viable cells of MII and rises above the surface. The apical cells of the aerial hyphae differentiate into spores that can spread in the environment 


\section{Peptidoglycan hydrolases}

The family of peptidoglycan (PG) hydrolases is composed of a large heterogeneous group of enzymes that assembly, modify and degrade PG, the major component of the cell envelope whose integrity is essential to bacterial survival. ${ }^{65,114} \mathrm{PG}$ hydrolases are present in eukaryotic and prokaryotic organisms as well as in phages. In animals and plants, they are involved in the defence against bacterial infections as a part of the innate immunity. ${ }^{115}$ In phages, they contribute to the mechanism of infection of bacteria. ${ }^{63,64}$

In bacteria, they are implicated in several physiological functions: cell wall turnover, cell separation, formation of flagella and pili, sporulation, and spore germination. Moreover, they are essential for the bacterial population being involved in developmental lysis, biofilm formation and autolysis in: genetic transformation, unfavourable growth conditions, and under exposure of antibiotics. ${ }^{7,33,65}$

$P G$ hydrolases are classified on the specificity of their cleavage sites. ${ }^{65} \mathrm{PG}$ amidases, cleave the amide bond between $\mathrm{N}$-acetyl muramic acid and L-alanine. PG peptidases cleave peptide bonds within the peptides (endopeptidases) or remove C-terminal amino acids (carboxypeptidases). Both are further classified in DD-, LD- and DL-peptidases depending on the amino acids involved in the cleavage. The PG hydolases family also includes a group of enzymes ( $N$-acetylmuramidases, lysozymes and lytic transglycosylases) that cleave the glycosidic bond between $\mathrm{N}$-acetyl muramic acid and $\mathrm{N}$-acetyl glucosamine. Finally, $\mathrm{N}$-acetylglucosaminidases hydrolyse the glycosidic bond between $\mathrm{N}$-acetylglucosamine and the neighbouring monosaccharides. This last enzyme is less specific and is involved in cleavage within oligosaccharides' different from PG.

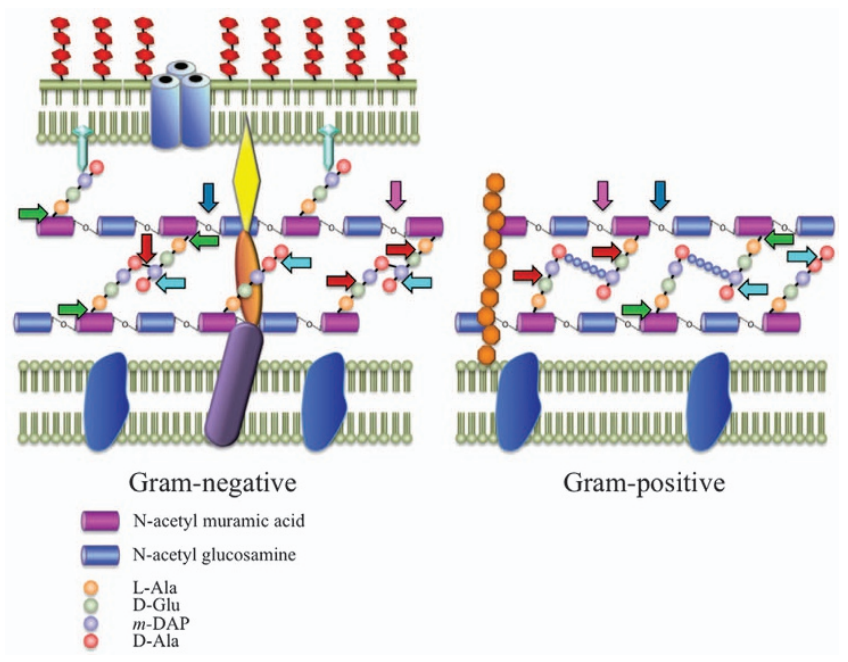

Figure Box. Schematic representation of Gram-positive and Gram-negative bacterial cell envelopes showing the cleavage specificities of PG hydrolases. PG amidases (green arrows); Endopeptidases (red arrows); Carboxypeptidases (light blue arrows); $\mathrm{N}$-acetylmuramidases (blue arrows); $\mathrm{N}$-acetylglucosaminidases (magenta arrows) mDAP: meso-diaminopimelic acid.

component and it mediates the release of DNA from sensitive streptococci. CbpD also activates two PG hydrolases (Box 1), LytA and LytC, resulting in increased lysis of susceptible cells. The process involves also two bacteriocins (CibA and CibB) that act by forming pores in the cytoplasmic membrane. ${ }^{36,68}$ In addition, lysis of the killed cells causes the release of pneumolysin, a key virulent factor that contributes to the pathogenesis of the pneumococcal disease in humans. ${ }^{36}$

Autolysis in M. xanthus development. $M$. xanthus is a free-living Gram-negative bacterium commonly found in soil with a complex life cycle that includes vegetative growth and a developmental pathway ${ }^{34}$ (Figure 2c). Under nutritional stress, the majority of bacteria aggregate to form a bulging mass called fruiting body while a small number of vegetative cells (about $10 \%$ ), named peripheral rods, remain undifferentiated and are located around and between fruiting bodies. ${ }^{69,70}$ Within the interior of the fruiting bodies, Mixobacteria differentiate into spores that are then released into the environment. During the early steps of development of the fruiting bodies, up to $90 \%$ of cells undergo altruistic cell lysis releasing their content which feeds the remaining cells that will differentiate into myxospores. ${ }^{34,65}$ Although $M$. xanthus contains several autolysin genes encoding for $P G$ hydrolases (Box 1), the pathway leading to autolysis is yet not 
well characterized. ${ }^{28,71}$ In some strains, a type II TA system, MazF/MrpC, seems to be involved in cell lysis during the fruiting body formation. ${ }^{71}$ Before sporulation, the degradation of MrpC by cell proteases causes the activation of the toxin MazF resulting in autolysis. ${ }^{71}$ However, in other $M$. xanthus strains, MazF is not essential for PCD or sporulation, ${ }^{72}$ therefore it appears that two parallel, potentially redundant pathways leading to $\mathrm{PCD}$ are present in different strains. ${ }^{73}$

Streptomyces developmental cycle. Streptomyces are filamentous soil bacteria, known for the production of several bioactive compounds such as antibiotics. They have a complex developmental cycle characterized by multicellular behaviour and mycelial growth. ${ }^{35,74}$ In vitro, their life cycle shows the formation of two differentiated structures: a vegetative substrate mycelium and a reproductive aerial mycelium. $^{74}$ In Streptomyces genus cycle, a part of the mycelium dies through a highly ordered process of PCD that occurs in two phases: during development of the vegetative mycelium and before sporulation (Figure 2d). ${ }^{35,75,76}$ In solid culture, the vegetative mycelium forms when Streptomyces grow on the agar surface forming compartmentalized mycelium (MI, first mycelium), which originates from the germination of a dormant spore when it encounters a permissive environment. Under stress conditions, such as nutrient depletion, a part of Ml cells is subjected to PCD while the remaining viable cells differentiate into multinucleated mycelium (MII, second mycelium). Multinucleated mycelium would favour rapid growth and nucleoid division, crucial for sporulation. ${ }^{35}$ At this stage, Streptomyces Mll cells are subjected to a new phase of PCD. A part of vegetative mycelium dies releasing nutrients to feed the aerial mycelium. The aerial hyphae are developed from branches of the remaining viable cells of $\mathrm{MII}$ and rise above the surface. Simultaneously, a non-growing part of the colony synthesizes antibiotics through a secondary metabolism. Finally, the apical cells of the aerial hyphae differentiate into hydrophobic spores that can spread the bacteria in the environment. ${ }^{74}$

When PCD occurs, the mycelium is subjected to progressive cell disorganization coupled to DNA degradation and followed by loss of the cell wall and membrane integrity and release of the intracellular content into the environment. ${ }^{76}$ It has been observed that proteins involved in the cellular disassembly, such as hydrolases, proteases, and enzymes implicated in membrane degradation, are produced during PCD in Streptomyces. ${ }^{77}$ The biological significance of PCD in Streptomyces colonies is still unclear, but it is probable that PCD is necessary to provide nutrients for further developmental processes such as sporulation and the production of antibiotics to protect the colony from neighbouring microorganisms. It has been also speculated that in these microorganisms PCD may be involved in competence, a process through which DNA fragments are incorporated in cells by transformation followed by recombination events obtaining a bundle of variable spores. ${ }^{35}$

Recently, it has been shown that PCD can be induced in Streptomyces lividans and $S$. coelicolor through a type II TA system belonging to the YefM/YoeB subfamily with high similarity to the one described in E. coli YefM/YoeB. ${ }^{78,79}$ As in $E$. coli, the toxin acts inhibiting translation initiation by processing the mRNA three bases downstream of the initiation codon. To date, the role of this TA system in Streptomyces PCD is still not clear. $^{78}$

Coccoid forms in Helicobacter pylori. The bacterium $H$. pylori is recognized as the main cause of gastric and duodenal ulcers and has a primary role in the development of gastric cancer. ${ }^{80,81}$ It can switch from the normal helical morphology into a coccoid-shaped resistant form when exposed to environmental stress, such as nutrients deprivation and a non-permissive temperature. In the coccoid population, a part of the cells enters a state of low metabolic activity and stops dividing (viable but non-culturable state), as a form of temporary adaption to an incompatible environment. Under these conditions, some of the coccoid cells undergo PCD, showing electron-dense bodies - probably deriving from DNA condensation - resembling micronuclei of apoptotic eukaryotic cells. ${ }^{80}$ This similarity is also supported by the identification of endonucleolytic DNA cleavage in these cells. ${ }^{80}$ It is supposed that $H$. pylori chooses to reduce its cell density through this mechanism to preserve itself and restart cell division in a suitable environment.

\section{Role of PCD in Biofilms}

Biofilms are structurally and dynamically complex biological systems. ${ }^{82,83}$ They are multicellular sessile communities characterized by cells embedded in a self-produced matrix of extracellular polymeric substances (EPS) and interspersed with open water channels. They exist both as mono- and multispecies communities. ${ }^{82,83}$ EPS include proteins, polysaccharides, and extracellular DNA (eDNA). Bacteria through biofilms can adhere to biological and non-biological surfaces, such as human tissues and medical implants. Indeed, biofilm formation has a role in several infectious diseases, including endocarditis, urinary tract and cystic fibrosis infections, and infections of artificial heart valves, joint prostheses, and catheters. $^{82}$ Furthermore, biofilms protect bacteria from stressing conditions as well as from other microorganisms that live in the same environment. Biofilms are associated with resistance to a broad range of antimicrobial agents, contributing to resistance to antibiotic treatments. ${ }^{7}$ Spore-forming bacteria produce both biofilm and endospores being able to respond more swiftly to environmental stresses. ${ }^{84}$ Moreover, biofilm is an optimal environment for sporulation. ${ }^{84}$

The control of bacterial cell death and lysis is supposed to be an important mechanism in cell differentiation and development of bacteria inside biofilms as well as for diffusion of bacteria into the environment. ${ }^{85,86}$ For example, under starvation a part of cells inside a biofilm autolyse providing nutrients for the remaining bacteria allowing them to colonize new sites (Figure 3). ${ }^{85}$ To release and disperse cells from biofilm into the environment, bacteria adopt at least three different mechanisms: erosion, sloughing, and seeding. ${ }^{87}$ Erosion consists of the continuous release of single or small group of cells during biofilm formation. Sloughing indicates cell aggregates that are shed from biofilm, usually during its later stages of formation. Seeding dispersal is an active process in which a large number of individual cells are released from hollow cavities formed inside the biofilm. ${ }^{87}$ In addition, eDNA, 


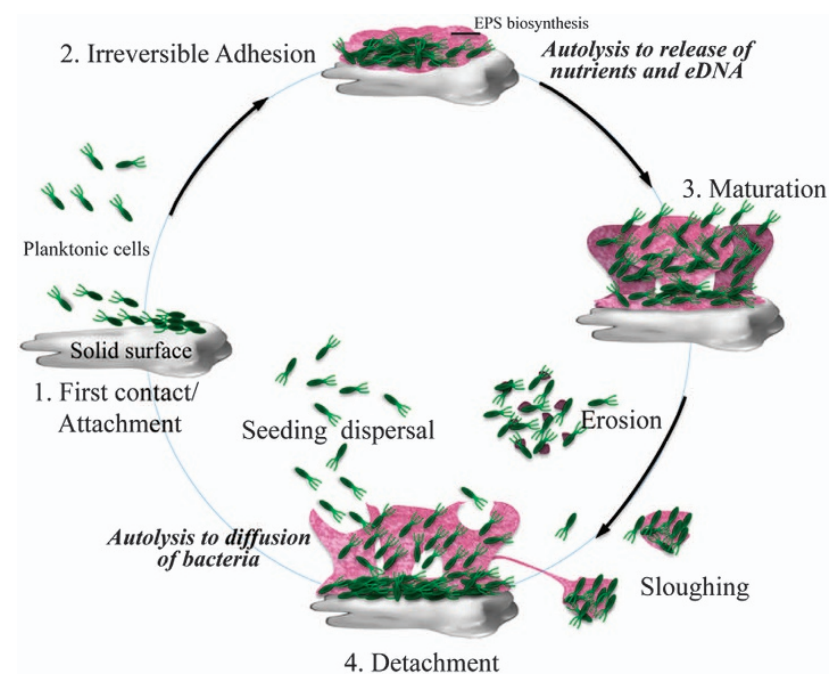

Figure 3 Biofilm formation and development. Initially, planktonic cells adhere to a solid surface (1), and production of extracellular polymeric substances (EPS) stabilizes the adhered colony (2). Some of the cells undergo autolysis releasing nutrients and eDNA that promote growth and maturation of the biofilm (3). Cells are dispersed from the biofilm and can colonise other sites through three mechanisms: erosion, sloughing, and seeding dispersal (4). Seeding dispersal implicates an active process of autolysis resulting in release of single bacterial cells and cavity formation

an essential component of the biofilm matrix, is released during biofilm development by cell lysis of a part of the bacterial population. eDNA contributes to the stability and development of the biofilm by binding to other EPS biopolymers. Moreover, through an acid-base interaction, it promotes adhesion between cells and between cells and surfaces. ${ }^{88-91}$ Several cell lysis factors such as autolysins and prophages contribute to eDNA release. ${ }^{90,91}$ Extracellular DNA release is mediated by QS mechanisms in both Gram-negative and Gram-positive bacteria. ${ }^{90,91}$ In the Gram-negative $P$. aeruginosa, QS molecules control the production of cell lysis factors such as prophages and phenazines (through the production of reactive oxygen species) that induce cell lysis causing eDNA release. $^{90}$ In the Gram-positive S. epidermidis, the agr QS system regulates the expression of the autolysin AtIE, resulting in cell lysis and eDNA release. ${ }^{92}$

PCD has been shown to occur in biofilm in both Grampositives and Gram-negatives through a number of mechanisms. ${ }^{53,54,86,93-97}$

In $S$. aureus, cidABC and IrgAB operons regulate autolysis and eDNA release by controlling $P G$ hydrolase activity through CidA and LrgA, proteins analogous to bacteriophage holins and antiholins. ${ }^{86,93}$

$P$. aeruginosa is involved in chronic infections of the respiratory tract such as cystic fibrosis, a notable biofilmbased disease. ${ }^{98-100}$ In $P$. aeruginosa, biofilm develops in a multi-step cellular cycle that is initiated by the attachment of free cells to a surface, followed by formation and maturation of multicellular structures (microcolonies), and finally by an active dispersal mechanism that spreads bacteria resulting in occupation of new surfaces. At this stage, cell death has been observed in a subpopulation of cells within the microcolonies. ${ }^{96,101}$ Dispersal and cell death events have been associated with the conversion of a genomic prophage to an infective lytic form. ${ }^{96}$ The action of lytic phage is correlated with the appearance inside microcolonies of phenotypic and functional variants, through transfer of genetic material, that spread and colonize new surfaces. ${ }^{101}$ Finally, in biofilm formation by Streptococcus mutans ${ }^{95}$ a QS system activates a death programme. Indeed, in this species when high density is reached a pherormone known as CSP is produced that induces upregulation of an autolysis effector, the CipB bacteriocin.

\section{Conclusions}

Similarly to eukaryotes, PCD in bacteria is a complex and regulated process that contributes to multiple aspects of bacterial communities' survival, differentiation, and spreading. The similarities observed between cell death systems of animals, plants, and bacteria suggests a common origin and presumably is a consequence of the endosymbiotic acquisition of bacteria by eukaryotes. ${ }^{3,102-104}$ According to this theory, aerobic and phototrophic bacteria, ingested by preeukaryotic cells, have evolved into mitochondria and chloroplasts, respectively. As previously reported, $S$. aureus CidA and LrgA proteins, located in the cytoplasmic membrane, exhibit bacteriophage holin-antiholin-like properties. ${ }^{86}$ The primary components of $\mathrm{PCD}$ regulatory control in animals, the mitochondria-associated Bax/Bcl-2 family of proteins, ${ }^{1,105-107}$ also display molecular and functional similarity to holin/ antiholin proteins supporting this theory. ${ }^{86}$ According to this idea, different organisms would use common strategies to control PCD and supposedly unlinked proteins could be evolutionarily related. ${ }^{86,102}$ However, although phylogenetically conserved PCD-related genes are clearly present in Eukarya branch of the tree of life, ${ }^{3}$ no evidence for such a presence exists in the Bacteria and Archaea branches. Furthermore, the presence of these genes or even their partial similarity with some eukaryote genes could be attributable to phenomena, such as genetic convergence or horizontal gene transfer. A thorough description of the evolution of PCD in bacteria is, however, beyond the scope of this review.

Studies on bacterial PCD are of great interest as it can be exploited to develop efficient alternative therapeutic strategies against bacteria resistant to current antibiotics. ${ }^{108}$ In fact, antimicrobial resistance in bacteria is on the rise and represents a worldwide emergency, and the number of antibiotics that retain activity against several serious pathogens is limited. ${ }^{109,110}$ This problem has been further amplified by the shortage of new molecules and requires novel approaches to the problem. Promising results have been obtained exploiting TA systems as antibacterial strategies via artificial PCD activation. Cell death is obtained by disruption or arrest of TA system formation or increased degradation of antitoxin. ${ }^{19,111}$

However, it should also be kept in mind that PCD induction could be damaging to the host when it is coupled with the release of extracellular products. Indeed, in some cases the activation of PCD can induce an increase of extracellular products that favour the pathogens. ${ }^{108}$

Finally, various studies indicate QS system as an attractive alternative approach to antibiotic therapy. ${ }^{49,112,113}$ In fact, QS 
systems are involved in the regulation of virulence factors in bacteria, and their inhibition could increase the susceptibility of the pathogens to the host defences.

Additional investigations - such as in vivo studies - are required to evaluate the real potentiality of these new therapies.

\section{Conflict of Interest}

The authors declare no conflict of interest.

Acknowledgements. This work was partially supported by the Italian 'Ministero per l'Istruzione, l'Università e la Ricerca' (MIUR).

1. Favaloro B, Allocati N, Graziano V, Di llio C, De Laurenzi V. Role of apoptosis in disease. Aging (Albany NY) 2012; 4: 330-349.

2. Green DR, Levine $B$. To be or not to be? how selective autophagy and cell death govern cell fate. Cell 2014; 157: 65-75.

3. Ameisen JC. On the origin, evolution, and nature of programmed cell death: a timeline of four billion years. Cell Death Differ 2002; 9: 367-393.

4. Knight RA, Melino G. Cell death in disease: from 2010 onwards. Cell Death Dis 2011; 2: e202.

5. Hill JH, Baehrecke EH. Autophagy in nonmammalian systems In: Melino G, Vaux D (eds) Cell Death. John Wiley \& Sons, Ltd: Chichester, UK, 2010, pp 189-197.

6. Bozhkov PV, Lam E. Green death: revealing programmed cell death in plants. Cell Death Differ 2011; 18: 1239-1240.

7. Rice KC, Bayles KW. Molecular control of bacterial death and lysis. Microbiol Mol Biol Rev 2008; 72: 85-109.

8. Bagchi S, Oniku AE, Topping K, Mamhoud ZN, Paget TA. Programmed cell death in Giardia. Parasitology 2012; 139: 894-903.

9. Sharon A, Finkelstein A, Shlezinger N, Hatam I. Fungal apoptosis: function, genes and gene function. FEMS Microbiol Rev 2009; 33: 833-854.

10. Gordeeva AV, Labas YA, Zvyagilskaya RA. Apoptosis in unicellular organisms: mechanisms and evolution. Biochemistry (Mosc) 2004; 69: 1055-1066.

11. Ning SB, Guo HL, Wang L, Song YC. Salt stress induces programmed cell death in prokaryotic organism Anabaena. J Appl Microbiol 2002; 93: 15-28.

12. Wang YS, Wang ZY. Sodium citrate induces apoptosis in biocontrol yeast Cryptococcus laurentii. J Appl Microbiol 2012; 113: 135-142.

13. Chowdhury S, Mukherjee T, Chowdhury SR, Sengupta S, Mukhopadhyay S, Jaisankar P et al. Disuccinyl betulin triggers metacaspase dependent endonuclease $\mathrm{G}$ mediated cell death in unicellular protozoan parasite Leishmania donovani. Antimicrob Agents Chemother 2014 58: 2186-2201

14. Bayles KW. Bacterial programmed cell death: making sense of a paradox. Nat Rev Microbiol 2014; 12: 63-69.

15. Claessen D, Rozen DE, Kuipers OP, Sogaard-Andersen L, van Wezel GP. Bacterial solutions to multicellularity: a tale of biofilms, filaments and fruiting bodies. Nat Rev Microbiol 2014; 12: 115-124.

16. Melino G. The Sirens' song. Nature 2001; 412: 23

17. Markx GH, Andrews JS, Mason VP. Towards microbial tissue engineering? Trends Biotechnol 2004; 22: 417-422.

18. Padmanabhan PK, Samant M, Cloutier S, Simard MJ, Papadopoulou B. Apoptosis-like programmed cell death induces antisense ribosomal RNA (rRNA) fragmentation and rRNA degradation in Leishmania. Cell Death Differ 2012; 19: 1972-1982.

19. Engelberg-Kulka H, Sat B, Reches M, Amitai S, Hazan R. Bacterial programmed cell death systems as targets for antibiotics. Trends Microbiol 2004; 12: 66-71.

20. Ng WL, Bassler BL. Bacterial quorum-sensing network architectures. Annu Rev Genet 2009; 43: 197-222.

21. Rutherford ST, Bassler BL. Bacterial quorum sensing: its role in virulence and possibilities for its control. Cold Spring Harb Perspect Med 2012; 2: a012427.

22. Solano C, Echeverz M, Lasa I. Biofilm dispersion and quorum sensing. Curr Opin Microbiol 2014; 18: 96-104.

23. Melino G, Knight RA, Nicotera P. How many ways to die? How many different models of cell death? Cell Death Differ 2005; 12: 1457-1462.

24. Galluzzi L, Vitale I, Abrams JM, Alnemri ES, Baehrecke EH, Blagosklonny MV et al. Molecular definitions of cell death subroutines: recommendations of the Nomenclature Committee on Cell Death 2012. Cell Death Differ 2012; 19: 107-120.

25. Vanlangenakker N, Vanden Berghe $\mathrm{T}$, Vandenabeele P. Many stimuli pull the necrotic trigger, an overview. Cell Death Differ 2012; 19: 75-86.

26. Suzanne M, Steller H. Shaping organisms with apoptosis. Cell Death Differ 2013; 20 669-675.

27. Chaloupka J, Vinter V. Programmed cell death in bacteria. Folia Microbiol 1996; 41: 451-464.

28. Lewis K. Programmed death in bacteria. Microbiol Mol Biol Rev 2000; 64: 503-514

29. Yamaguchi $\mathrm{Y}$, Park JH, Inouye M. Toxin-antitoxin systems in bacteria and archaea. Annu Rev Genet 2011; 45: 61-79.
30. Zheng W, Rasmussen U, Zheng S, Bao X, Chen B, Gao Y et al. Multiple modes of cell death discovered in a prokaryotic (cyanobacterial) endosymbiont. PLOS One 2013; 8: e66147.

31. Tanouchi Y, Pai A, Buchler NE, You L. Programming stress-induced altruistic death in engineered bacteria. Mol Syst Biol 2012; 8: 626 .

32. Schuster CF, Bertram R. Toxin-antitoxin systems are ubiquitous and versatile modulators of prokaryotic cell fate. FEMS Microbiol Lett 2013; 340: 73-85.

33. Dwyer DJ, Camacho DM, Kohanski MA, Callura JM, Collins JJ. Antibiotic-induced bacterial cell death exhibits physiological and biochemical hallmarks of apoptosis. Mol Cell 2012; 46: $561-572$.

34. Wireman JW, Dworkin M. Developmentally induced autolysis during fruiting body formation by Myxococcus xanthus. J Bacteriol 1977; 129: 798-802.

35. Yagüe P, López-García MT, Rioseras B, Sánchez J, Manteca A. Pre-sporulation stages of Streptomyces differentiation: state-of-the-art and future perspectives. FEMS Microbiol Lett 2013; 342: 79-88.

36. Claverys JP, Havarstein LS. Cannibalism and fratricide: mechanisms and raisons d'etre. Nat Rev Microbiol 2007; 5: 219-229.

37. Van Melderen L. Toxin-antitoxin systems: why so many, what for? Curr Opin Microbiol 2010; 13: $781-785$.

38. Wen Y, Behiels E, Devreese B. Toxin-antitoxin systems: their role in persistence, biofilm formation and pathogenicity. Pathog Dis 2014; 70: 240-249.

39. Park SJ, Son WS, Lee BJ. Structural overview of toxin-antitoxin systems in infectious bacteria: a target for developing antimicrobial agents. Biochim Biophys Acta 2013; 1834: 1155-1167.

40. Mruk I, Kobayashi I. To be or not to be: regulation of restriction-modification systems and other toxin-antitoxin systems. Nucleic Acids Res 2014; 42: 70-86.

41. Fozo EM, Makarova KS, Shabalina SA, Yutin N, Koonin EV, Storz G. Abundance of type I toxin-antitoxin systems in bacteria: searches for new candidates and discovery of novel families. Nucleic Acids Res 2010; 38: 3743-3759.

42. Unoson C, Wagner EG. A small SOS-induced toxin is targeted against the inner membrane in Escherichia coli. Mol Microbiol 2008; 70: 258-270.

43. Tan Q, Awano N, Inouye M. YeeV is an Escherichia coli toxin that inhibits cell division by targeting the cytoskeleton proteins, FtsZ and MreB. Mol Microbiol 2011; 79: 109-118.

44. Schmidt O, Schuenemann VJ, Hand NJ, Silhavy TJ, Martin J, Lupas AN et al. prIF and yhaV encode a new toxin-antitoxin system in Escherichia coli. J Mol Biol 2007; 372: 894-905.

45. Engelberg-Kulka H, Amitai S, Kolodkin-Gal I, Hazan R. Bacterial programmed cell death and multicellular behavior in bacteria. PLOS Genet 2006; 2: e135.

46. Engelberg-Kulka H, Hazan R, Amitai S. mazEF: a chromosomal toxin-antitoxin module that triggers programmed cell death in bacteria. J Cell Sci 2005; 118: 4327-4332.

47. Belitsky M, Avshalom H, Erental A, Yelin I, Kumar S, London N et al. The Escherichia coli extracellular death factor EDF induces the endoribonucleolytic activities of the toxins MazF and ChpBK. Mol Cell 2011; 41: 625-635.

48. Kumar S, Kolodkin-Gal I, Engelberg-Kulka H. Novel quorum-sensing peptides mediating interspecies bacterial cell death. MBio 2013; 4: e00314-00313.

49. Kumar S, Engelberg-Kulka H. Quorum sensing peptides mediating interspecies bacteria cell death as a novel class of antimicrobial agents. Curr Opin Microbiol 2014; 21C: 22-27.

50. Mutschler H, Gebhardt M, Shoeman RL, Meinhart A. A novel mechanism of programmed cell death in bacteria by toxin-antitoxin systems corrupts peptidoglycan synthesis. PLoS Biol 2011; 9: e1001033.

51. Gerdes K, Maisonneuve E. Bacterial persistence and toxin-antitoxin loci. Annu Rev Microbiol 2012; 66: 103-123.

52. Schumacher MA, Piro KM, Xu W, Hansen S, Lewis K, Brennan RG. Molecular mechanisms of HipA-mediated multidrug tolerance and its neutralization by HipB. Science 2009; 323 : 396-401.

53. Yamaguchi $Y$, Inouye M. Regulation of growth and death in Escherichia coli by toxinantitoxin systems. Nat Rev Microbiol 2011; 9: 779-790.

54. Wang X, Wood TK. Toxin-antitoxin systems influence biofilm and persister cell formation and the general stress response. Appl Environ Microbiol 2011; 77: 5577-5583.

55. Fineran PC, Blower TR, Foulds IJ, Humphreys DP, Lilley KS, Salmond GP. The phage abortive infection system, ToxIN, functions as a protein-RNA toxin-antitoxin pair. Proc Natl Acad Sci USA 2009; 106: 894-899.

56. Blower TR, Fineran PC, Johnson MJ, Toth IK, Humphreys DP, Salmond GP. Mutagenesis and functional characterization of the RNA and protein components of the toxIN abortive infection and toxin-antitoxin locus of Erwinia. J Bacteriol 2009; 191 6029-6039.

57. Labrie SJ, Samson JE, Moineau S. Bacteriophage resistance mechanisms. Nat Rev Microbiol 2010; 8: 317-327.

58. Samson JE, Spinelli S, Cambillau C, Moineau S. Structure and activity of AbiQ, a lactococcal endoribonuclease belonging to the type III toxin-antitoxin system. Mol Microbiol 2013: 87: 756-768.

59. Blower TR, Short FL, Rao F, Mizuguchi K, Pei XY, Fineran PC et al. Identification and classification of bacterial Type III toxin-antitoxin systems encoded in chromosomal and plasmid genomes. Nucleic Acids Res 2012; 40: 6158-6173.

60. Cook GM, Robson JR, Frampton RA, McKenzie J, Przybilski R, Fineran PC et al. Ribonucleases in bacterial toxin-antitoxin systems. Biochim Biophys Acta 2013; 1829: 523-531. 
61. Masuda H, Tan Q, Awano N, Wu KP, Inouye M. YeeU enhances the bundling of cytoskeletal polymers of MreB and FtsZ, antagonizing the $\mathrm{CbtA}(\mathrm{YeeV})$ toxicity in Escherichia coli. Mol Microbiol 2012; 84: 979-989.

62. Wang X, Lord DM, Cheng HY, Osbourne DO, Hong SH, Sanchez-Torres V et al. A new type $\mathrm{V}$ toxin-antitoxin system where mRNA for toxin GhoT is cleaved by antitoxin GhoS. Nat Chem Biol 2012; 8: 855-861.

63. Catalao MJ, Gil F, Moniz-Pereira J, Sao-Jose C, Pimentel M. Diversity in bacterial lysis systems: bacteriophages show the way. FEMS Microbiol Rev 2013; 37: 554-571.

64. Borysowski J, Weber-Dabrowska B, Gorski A. Bacteriophage endolysins as a novel class of antibacterial agents. Exp Biol Med (Maywood) 2006; 231: 366-377.

65. Vollmer W, Joris B, Charlier P, Foster S. Bacterial peptidoglycan (murein) hydrolases. FEMS Microbiol Rev 2008; 32: 259-286.

66. Gonzalez-Pastor JE. Cannibalism: a social behavior in sporulating Bacillus subtilis. FEMS Microbiol Rev 2011; 35: 415-424.

67. Lamsa A, Liu WT, Dorrestein PC, Pogliano K. The Bacillus subtilis cannibalism toxin SDP collapses the proton motive force and induces autolysis. Mol Microbiol 2012; 84: 486-500.

68. Wei H, Havarstein LS. Fratricide is essential for efficient gene transfer between pneumococci in biofilms. Appl Environ Microbiol 2012; 78: 5897-5905.

69. O'Connor KA, Zusman DR. Behavior of peripheral rods and their role in the life cycle of Myxococcus xanthus. J Bacteriol 1991; 173: 3342-3355.

70. Zusman DR, Scott AE, Yang Z, Kirby JR. Chemosensory pathways, motility and development in Myxococcus xanthus. Nat Rev Microbiol 2007; 5: 862-872.

71. Nariya $\mathrm{H}$, Inouye M. MazF, an mRNA interferase, mediates programmed cell death during multicellular Myxococcus development. Cell 2008; 132: 55-66.

72. Boynton TO, McMurry JL, Shimkets LJ. Characterization of Myxococcus xanthus MazF and implications for a new point of regulation. Mol Microbiol 2013; 87: 1267-1276.

73. Lee B, Holkenbrink C, Treuner-Lange A, Higgs PI. Myxococcus xanthus developmental cell fate production: heterogeneous accumulation of developmental regulatory proteins and reexamination of the role of MazF in developmental lysis. J Bacteriol 2012; 194: 3058-3068.

74. McCormick JR, Flardh K. Signals and regulators that govern Streptomyces development. FEMS Microbiol Rev 2012; 36: 206-231.

75. Miguelez EM, Hardisson C, Manzanal MB. Hyphal death during colony development in Streptomyces antibioticus: morphological evidence for the existence of a process of cell deletion in a multicellular prokaryote. J Cell Biol 1999; 145: 515-525.

76. Manteca A, Fernandez M, Sanchez J. Cytological and biochemical evidence for an early cell dismantling event in surface cultures of Streptomyces antibioticus. Res Microbiol 2006; 157: $143-152$.

77. Manteca A, Mader U, Connolly BA, Sanchez J. A proteomic analysis of Streptomyces coelicolor programmed cell death. Proteomics 2006; 6: 6008-6022.

78. Sevillano L, Diaz M, Yamaguchi Y, Inouye M, Santamaria RI. Identification of the first functional toxin-antitoxin system in Streptomyces. PLoS One 2012; 7: e32977.

79. Kamada K, Hanaoka F. Conformational change in the catalytic site of the ribonuclease YoeB toxin by YefM antitoxin. Mol Cell 2005; 19: 497-509.

80. Cellini L, Robuffo I, Maraldi NM, Donelli G. Searching the point of no return in Helicobacter pylori life: necrosis and/or programmed death? J Appl Microbiol 2001; 90: 727-732.

81. Sibony M, Jones NL. Recent advances in Helicobacter pylori pathogenesis. Curr Opin Gastroenterol 2012; 28: 30-35.

82. Hall-Stoodley L, Costerton JW, Stoodley P. Bacterial biofilms: from the natural environment to infectious diseases. Nat Rev Microbiol 2004; 2: 95-108.

83. Elias S, Banin E. Multi-species biofilms: living with friendly neighbors. FEMS Microbiol Rev 2012; 36: 990-1004.

84. Obana N, Nakamura K, Nomura N. A sporulation factor is involved in morphological change of Clostridium perfringens biofilm in response to temperature. J Bacteriol 2014; 196: 1540-1550.

85. Webb JS, Givskov M, Kjelleberg S. Bacterial biofilms: prokaryotic adventures in multicellularity. Curr Opin Microbiol 2003; 6: 578-585.

86. Bayles KW. The biological role of death and lysis in biofilm development. Nat Rev Microbiol 2007; 5: 721-726

87. Kaplan JB. Biofilm dispersal: mechanisms, clinical implications, and potential therapeutic uses. J Dent Res 2010; 89: 205-218.

88. Grande R, Di Giulio M, Bessa LJ, Di Campli E, Baffoni M, Guarnieri S et al. Extracellular DNA in Helicobacter pylori biofilm: a backstairs rumour. J Appl Microbiol 2011; 110: 490-498.

89. Rice KC, Mann EE, Endres JL, Weiss EC, Cassat JE, Smeltzer MS et al. The cidA murein hydrolase regulator contributes to DNA release and biofilm development in Staphylococcus aureus. Proc Natl Acad Sci USA 2007; 104: 8113-8118.

90. Das T, Sehar S, Manefield M. The roles of extracellular DNA in the structural integrity of extracellular polymeric substance and bacterial biofilm development. Environ Microbiol Rep 2013; 5: 778-786.
91. Montanaro L, Poggi A, Visai L, Ravaioli S, Campoccia D, Speziale $P$ et al. Extracellular DNA in biofilms. Int J Artif Organs 2011; 34: 824-831.

92. Qin Z, Ou Y, Yang L, Zhu Y, Tolker-Nielsen T, Molin S et al. Role of autolysin-mediated DNA release in biofilm formation of Staphylococcus epidermidis. Microbiology 2007; 153 2083-2092.

93. Sadykov MR, Bayles KW. The control of death and lysis in staphylococcal biofilms: a coordination of physiological signals. Curr Opin Microbiol 2012; 15: 211-215.

94. Moormeier DE, Endres JL, Mann EE, Sadykov MR, Horswill AR, Rice KC et al. Use of microfluidic technology to analyze gene expression during Staphylococcus aureus biofilm formation reveals distinct physiological niches. Appl Environ Microbiol 2013; 79: 3413-3424.

95. Perry JA, Cvitkovitch DG, Levesque CM. Cell death in Streptococcus mutans biofilms: a link between CSP and extracellular DNA. FEMS Microbiol Lett 2009; 299: 261-266.

96. Webb JS, Thompson LS, James S, Charlton T, Tolker-Nielsen T, Koch B et al. Cell death in Pseudomonas aeruginosa biofilm development. J Bacteriol 2003; 185: 4585-4592.

97. Ma L, Conover M, Lu H, Parsek MR, Bayles K, Wozniak DJ. Assembly and development of the Pseudomonas aeruginosa biofilm matrix. PLoS Pathog 2009; 5: e1000354.

98. Woo JK, Webb JS, Kirov SM, Kjelleberg S, Rice SA. Biofilm dispersal cells of a cystic fibrosis Pseudomonas aeruginosa isolate exhibit variability in functional traits likely to contribute to persistent infection. FEMS Immunol Med Microbiol 2012; 66: 251-264.

99. Rybtke MT, Jensen PO, Hoiby N, Givskov M, Tolker-Nielsen T, Bjarnsholt T. The implication of Pseudomonas aeruginosa biofilms in infections. Inflamm Allergy Drug Targets 2011; 10: 141-157.

100. Webb JS, Lau M, Kjelleberg S. Bacteriophage and phenotypic variation in Pseudomonas aeruginosa biofilm development. J Bacteriol 2004; 186: 8066-8073.

101. Kirov SM, Webb JS, O'May C Y, Reid DW, Woo JK, Rice SA et al. Biofilm differentiation and dispersal in mucoid Pseudomonas aeruginosa isolates from patients with cystic fibrosis. Microbiology 2007; 153: 3264-3274.

102. Wang J, Bayles KW. Programmed cell death in plants: lessons from bacteria? Trends Plant Sci 2013; 18: 133-139.

103. Koonin EV, Aravind L. Origin and evolution of eukaryotic apoptosis: the bacterial connection. Cell Death Differ 2002; 9: 394-404.

104. James ER, Green DR. Infection and the origins of apoptosis. Cell Death Differ 2002; 9: 355-357.

105. Galluzzi L, Kepp O, Kroemer G. Mitochondria: master regulators of danger signalling. Nat Rev Mol Cell Biol 2012; 13: 780-788.

106. Chipuk JE, Moldoveanu T, Llambi F, Parsons MJ, Green DR. The BCL-2 family reunion. Mol Cell 2010; 37: 299-310.

107. Czabotar PE, Lessene G, Strasser A, Adams JM. Control of apoptosis by the BCL-2 protein family: implications for physiology and therapy. Nat Rev Mol Cell Biol 2014; 15: 49-63.

108. Tanouchi Y, Lee AJ, Meredith H, You L. Programmed cell death in bacteria and implications for antibiotic therapy. Trends Microbiol 2013; 21: 265-270.

109. Nikaido H. Multidrug resistance in bacteria. Annu Rev Biochem 2009; 78: 119-146.

110. Allocati N, Masulli M, Alexeyev MF, Di llio C. Escherichia coli in Europe: an overview. Int J Environ Res Public Health 2013; 10: 6235-6254.

111. Williams JJ, Hergenrother PJ. Artificial activation of toxin-antitoxin systems as an antibacterial strategy. Trends Microbiol 2012; 20: 291-298.

112. El-Mowafy SA, Shaaban MI, Abd El Galil KH. Sodium ascorbate as a quorum sensing inhibitor of Pseudomonas aeruginosa. J Appl Microbiol 2014; 117: 1388-1399.

113. Bjarnsholt T, Givskov M. Quorum-sensing blockade as a strategy for enhancing host defences against bacterial pathogens. Philos Trans R Soc Lond B Biol Sci 2007; 362: 1213-1222.

114. Frirdich E, Gaynor EC. Peptidoglycan hydrolases, bacterial shape, and pathogenesis. Curr Opin Microbiol 2013; 16: 767-778.

115. Szweda P, Schielmann M, Kotlowski R, Gorczyca G, Zalewska M, Milewski S Peptidoglycan hydrolases-potential weapons against Staphylococcus aureus. Appl Microbiol Biotechnol 2012; 96: 1157-1174.



Cell Death and Disease is an open-access journal published by Nature Publishing Group. This work is licensed under a Creative Commons Attribution 4.0 International Licence. The images or other third party material in this article are included in the article's Creative Commons licence, unless indicated otherwise in the credit line; if the material is not included under the Creative Commons licence, users will need to obtain permission from the licence holder to reproduce the material. To view a copy of this licence, visit http://creativecommons.org/licenses/by/4.0 